\title{
Primum non nocere or primum facere meliorem? Hacking the brain in the 21st century
}

\author{
Primum non nocere ou primum facem meliorem? \\ O cérebro pirateado do século 21
}

Lucas Borrione, ${ }^{1}$ Andre R. Brunoni ${ }^{1,2}$

\begin{abstract}
Transcranial direct current stimulation (tDCS) is a non-invasive brain stimulation technique that modulates cortical excitability. It is devoid of serious adverse events and exerts variable effects on cognition, with several research findings suggesting that it can improve memory, verbal and mathematical skills. Because tDCS devices are low-cost, portable and relatively easy to assemble, they have become available outside of the medical setting and used for non-medical ("cosmetic") purposes by laypersons. In this sense, tDCS has become a popular technique aiming to improve cognition and the achievement of a better performance not only at work, but also in other fields such as sports, leisure activities (video games) and even the military. In spite of these unforeseen developments, there has been a general paralysis of the medical and regulatory agencies to develop guidelines for the use of tDCS for cosmetic purposes. Several challenges are present, most importantly, how to restrict tDCS use outside of the medical setting in face of variable and sometimes conflicting results from scientific research. This article aims to describe the popular use of tDCS, in light of the pillars of neuroethics, a branch of bioethics relative to brain research. Between two possible but extreme solutions - total release or total restriction of tDCS - it is paramount to develop a spectrum of alternatives, which may vary over time and in different cultural backgrounds.

Keywords: Transcranial direct current stimulation (tDCS); neuromodulation; neuroethics; bioethics; cosmetic cognitive enhancement.
\end{abstract}

\section{Resumo}

A estimulação transcraniana por corrente contínua (ETCC) é uma técnica não invasiva de estimulação cerebral que modula a excitabilidade cortical. A ETCC é desprovida de efeitos adversos graves e exerce efeitos variáveis sobre a cognição, com vários achados de pesquisa sugerindo que a técnica pode promover melhora nas habilidades mnêmica, verbal e matemática. Devido ao seu baixo custo, portabilidade e facilidade de montagem, os aparelhos de ETCC tornaram-se disponíveis fora do contexto médico, sendo usados para fins não médicos ("cosméticos") por indivíduos leigos. Nesse sentido, a ETCC tornou-se um procedimento popular para aprimoramento da cognição e a realização de melhor desempenho não somente no ambiente de trabalho, mas também em campos tais como o esporte, atividades de lazer (video games) e até no meio militar. Apesar desses acontecimentos imprevisíveis, há uma certa morosidade das agências médicas e regulatórias em desenvolver diretrizes para o uso de ETCC para fins cosméticos. Há muitos desafios presentes, principalmente, como restringir o uso da ETCC fora do contexto médico em face de resultados variáveis, e muitas vezes conflitantes, da pesquisa científica sobre o tema. Este artigo tem como objetivo descrever o uso popular da ETCC sob a luz da neuroética, um ramo da bioética que se dedica ao estudo do cérebro. Entre duas situações possíveis, mas extremas - liberação ou restrição totais da ETCC -, é primordial o desenvolvimento de um espectro de alternativas, que podem variar ao longo do tempo e depender de diversos contextos culturais.

Descritores: Estimulação transcraniana por corrente contínua (ETCC), neuromodulação, neuroética, bioética, aprimoramento cognitivo cosmético.

\footnotetext{
${ }^{1}$ Serviço Interdisciplinar de Neuromodulação, Laboratório de Neurociências (LIM-27), Departamento e Instituto de Psiquiatria (IPq), Hospital das Clínicas da Faculdade de Medicina da Universidade de São Paulo, São Paulo, Brazil. ${ }^{2}$ Department of Psychiatry and Psychotherapy, Ludwig-Maximilians-University, Munich, Germany.

Submitted Jun 06 2017, accepted for publication Aug 162017.

Suggested citation: Borrione L, Brunoni AR. Primum non nocere or primum facere meliorem? Hacking the brain in the 21st century. Trends Psychiatry Psychother. 2017;39(4):232-238. http://dx.doi.org/10.1590/2237-6089-2017-0075
} 


\section{Introduction}

Transcranial direct current stimulation (tDCS) is a noninvasive brain stimulation technique that, by means of low-intensity continuous electrical current applied to an intact scalp, modulates cortical excitability. ${ }^{1}$ tDCS seems to be devoid of serious side effects and able to induce therapeutic effects in varied mental disorders and neurological diseases, such as major depression and pain. ${ }^{2,3}$ Its low cost, simplicity and tolerability turn it into an attractive modality within the clinical setting. ${ }^{4}$

Despite its promising medical use, tDCS has been extensively used in several non-medical contexts, such as: 1) individuals leading a "do-it-yourself" tDCS (DIY-tDCS) movement over the Internet, displaying on their Youtube channels how to assemble tDCS devices and discussing on Reddit how they should be used ${ }^{5}$; 2) small or venture-backed, Silicon Valley startup companies commercializing tDCS for boosting memory, increasing athletic performance, inducing relaxation and improving playing electronic games ${ }^{6,7}$; and 3) American governmental agencies such as the Defense Advanced Research Projects Agency (DARPA), the innovation and technology agency affiliated with the U.S. Department of Defense, investigating whether the pro-cognitive effects of tDCS can be used to help scanning and targeting enemies in the theater of war. ${ }^{8}$

However, in spite of these unforeseen developments of tDCS research and practice, a general paralysis of medical and regulatory agencies has been observed. For instance, regulatory agencies such as the Food and Drug Administration (FDA) and the European Environmental Protection Agency (EPA) have presented no resolution regarding tDCS thus far, inasmuch as to decide whether tDCS is a "medical" device. ${ }^{9}$ In Brazil, similar idleness is observed in the Brazilian Federal Council of Medicine (Conselho Federal de Medicina - CFM) and the National Agency of Sanitary Surveillance (Agência Nacional de Vigilância Sanitária - ANVISA). Even though the latter has recently approved commercialization of a commercial device, tDCS use has not been broadly discussed. ${ }^{10}$

The aim of this article is to discuss tDCS use in the context of neuroethics, which is defined as "the study of ethical, legal and social issues that emerge when scientific findings about the brain change medical practices, legal interpretations, and health and social policies."11 Neuroethics investigates how the cardinal principles of bioethics (autonomy, non-maleficence, beneficence and justice) applies to the human brain, considering not only such unique ethical dilemmas, but also the consequences of using a brain intervention that can modify one's moral and ethical perspectives. We hereby discuss: potential tDCS effects on cognition and social behavior; ethical dilemmas, from a neuroethics perspective, regarding tDCS use; the regulatory status of tDCS around the world; and perspectives that can help decide whether and to which extent tDCS use should be regulated.

\section{tDCS effects in the context of neuroethics}

tDCS is used in neuropsychology to modify cognitive functions. For example, tDCS applied on the prefrontal cortex consistently increases cognitive performance in operational memory tests such as the n-back task. ${ }^{12}$ Sham-controlled studies applying tDCS on the posterior parietal cortex showed a change in visuospatial attention, creating an attentional bias of the hemineglect type, ${ }^{13}$ while others using tDCS over the Broca's area showed improvement in verbal fluency. ${ }^{14}$ Studies also showed that tDCS generated an improvement in the performance of mathematical tasks and retention and evocation of words. ${ }^{15,16}$

More complex cognitive functions, such as decisionmaking involving social and emotional aspects, are also modified by tDCS. For example, in a study investigating the feelings arising from social ostracism (using a game called Cyberball Task in which individuals "play" ball to each other, but do not "throw" it to the subject, who is left in ostracism), it was shown that tDCS increased the feelings of jealousy generated by ostracism. ${ }^{17}$ The effects of tDCS were also evaluated in the Ultimatum Game, a game in which a volunteer must decide whether to accept a part of someone else's money. If the person does not accept the proposal, both participants lose. Normally, volunteers do not accept very unfair proposals. However, a study showed that individuals who received tDCS on the medial prefrontal cortex were more likely to accept lower bids. ${ }^{18}$

Furthermore, tDCS can influence moral judgments. In a study in which individuals were presented to utilitarian dilemmas (illustrated by the Trolley Problem, in which an individual must decide whether to take a derailed train from the rails to save five people, however killing one person in the process, or else let the train on the rails, thereby killing all five people), anodal tDCS induced more non-utilitarian decisions. ${ }^{19}$ Also, tDCS can modify the ability of lying and cheating. In a study involving the use of tDCS to assess its influence on various types of lies, it specifically modulated deceptive responses to general information, leaving those on personal information unaffected. ${ }^{20}$

Nonetheless, even though most scientists have been relatively cautious in advertising the cognitive effects of 
tDCS, the general press has done so in a much more enthusiastic way. ${ }^{21}$ In fact, negative results were also observed. Some studies have even shown impairments in the performance of cognitive functions (notably those that have used cathodal stimulation over the area of interest), ${ }^{22}$ while others have proposed that tDCS leads to a trade-off in cognitive performance, improving one cognitive function at the expense of another, as well as inducing different effects according to the basal conditions of the individuals. For example, one study showed that tDCS improved performance in math tasks in individuals with high "math anxiety," but decreased performance in those with low "math anxiety," perhaps because of an overall effect on stress and anxiety reduction. ${ }^{23}$ Furthermore, recent meta-analyses have shown that the effects of tDCS on various cognitive functions are small - clinically irrelevant - or null.24,25 Finally, the ecological validity of tDCS effects, that is, its applicability to the "real world," is still unknown, since virtually all findings derive from experimental conditions in controlled environments. ${ }^{26}$

It should be underscored that the mechanisms of action of tDCS are not yet fully understood. Recent studies have shown that the direction of effects can be reversed according to the parameters applied (polarity, intensity and duration). ${ }^{27}$ The influence of parameters such as number of days of application and electrode size is still largely unknown, ${ }^{28}$ as are the effects of the interaction between tDCS and psychotropic agents, or the long-term effects of tDCS on adults, adolescents, children and the elderly. ${ }^{29,30}$

\section{tDCS safety}

No serious adverse events have been reported in thousands of patients that underwent tDCS so far; therefore, the technique is safe according to the parameters used in studies. ${ }^{31}$ In patients with mental disorders, however, the technique has not yet been sufficiently investigated, and cases of tDCS-induced mania or hypomania have been reported. . $^{32,33}$

Importantly, tDCS has been evaluated for safety in hospital/laboratory contexts. Its home use, as well as its "self-use" (DIY-tDCS), have been little investigated in the literature. For example, the tDCS application parameters tested are relatively restricted (e.g., currents of 1-2 mA, maximum of 30 minutes per day, and 20 sessions or less). Self-assembled devices do not contain security locks to prevent their use with different parameters. Even in controlled scenarios, few studies have evaluated the technique in children and adolescents. As the younger generations are more prone to incorporate new technologies, many DIY-tDCS users could be teenagers interested in improving their academic, game or sports performance.

Finally, although most companies specify that tDCS should only be used by healthy individuals, it is naïve to disregard that part of DIY-tDCS users could be individuals with mental disorders who, for a variety of reasons (lack of access to medical treatment, a wish for treatment immediacy or empowerment, or poor recognition of symptoms), do not seek medical help and prefer to try "self-treatment" or to "treat" a relative or a significant other following general advice provided by the Internet. The use of DIY-tDCS can delay the correct diagnosis and treatment of mental disorders, with harmful clinical consequences.

\section{Neuroethical dilemmas in the use of tDCS}

As tDCS devices are inexpensive, portable and easy to use and manufacture, their non-medical use has been increasingly widespread. In several countries, more and more individuals are purchasing tDCS devices over the Internet and using them according to tutorials offered by laypeople.

Moreover, the early availability of tDCS on the market for "cosmetic" reasons has generated important ethical concerns as, to date, there are no specific guidelines or indications for these purposes. At the same time, academics have presented several proposals for introducing specific regulatory measures, notably because of the limited knowledge about the long-term effects of the indiscriminate use of tDCS. ${ }^{9}$

In view of this unexpected context, the medical community is invited to take a stand against this new phenomenon, somewhat similar in its form to some types of recreational drug use, with all risks of abuse or dependence. Should practitioners stand against the non-medical use of tDCS, even if this is already a reality? Contrarily, would it be the best course of action to consent to the recreational use of this technique by adopting a "harm reduction" attitude, i.e., educating laypeople on its use and monitoring its abusive use and possible side effects?

Safety is the first ethical concern regarding DIY- or home-tDCS use. Here, the discussion shares similar points with that on the use of psychostimulants, such as methylphenidate and modafinil, by students or executives trying to improve performance. However, these drugs, unlike tDCS, are regulated and restricted.

Another concern is autonomy in deciding one's wish to receive tDCS. The U.S. military research agency 
DARPA, for example, has evaluated tDCS to improve the performance of soldiers in recognizing targets when monitoring, via satellite, war zones. ${ }^{8}$ There is also a theoretical concern to autonomy related to companies that sell tDCS devices for performance improvement in video games ${ }^{9,34}$; propose tDCS use for relaxation, sleep inducement and stress reduction ${ }^{9}$; or to improve the performance of athletes, including that of some American athletes in the recent 2016 edition of the Olympic Games.

If novel tDCS protocols prove to be more effective, there might be both implicit coercion (by social pressure) and explicit coercion (by the imposition of authority) towards its use. Implicit coercion, in this case, is closely related to some core values of Western societies, such as productivity and excellence. If pressures on productivity escalate, in this scenario, one may wonder about the actual "freedom of choice" of an individual regarding the use of tDCS for improvements in cognitive performance or memory. Furthermore, such coercion may become explicit if, for example, tDCS is used by parents to improve their children's school performance or by military services to improve soldiers' performance. Self-enhancement, if made standard, can undermine the individuals' freedom to choose whether to use tDCS.

Regarding justice, the widespread use of tDCS for cognitive enhancement can present a challenge for social and distributive justice, due to fear of the emergence of "neuro-enhanced" individuals. In this sense, Harari's book Homo Deus discusses that, as cognitive enhancement methods (neuroenhancers) are being developed on a larger scale, emerging concerns arise about their equitable distribution, which can be hampered by financial and social barriers, and potentially reinforce the gap between people with low and high purchasing power. ${ }^{35}$ tDCS use in competitive, "no pain, no gain" environments is also problematic, as cognitive enhancement can deepen already existing inequalities. ${ }^{36}$

Although tDCS devices are currently inexpensive, their price might increase due to more expensive handsets and/or monthly subscription services for updates and new programs. One possible solution, particularly in welfare states, would be to make tDCS use freely available to the broad population. However, the risk here is distorting health priorities, which is particularly relevant when only scarce health resources are available, raising the question of the legitimacy of using such resources for "cosmetic" purposes, mainly to improve cognitive performance in a context of uncertain risks and benefits.

Finally, there is the question of authenticity. Experiments with tDCS have shown that it can modify moral decisions. Memory modification can also be a menace to self-identity and the search for one's meaning of life, notably with respect to the standards of human excellence and happiness. This concern is relevant both at the individual level (personality changes) and at the collective level (changes of human behavior). In the individual level, the distinction between normal and pathological is very relevant. For example, the use of tDCS for improved attention can be perceived in different ways by a healthy individual, as opposed to one with attention deficit hyperactive disorder (ADHD). In the latter case, tDCS allows the individual to restore an impaired function as part of a clinical treatment; in healthy persons, tDCS can be used as a substitute for something that the individual could have acquired by other means, such as patience, persistence, and dedication.

\section{Regulatory aspects}

The use of tDCS in clinical research and practice is regulated by ethical procedures such as informed consent, an appraisal of the risk-benefit ratio (benefits should outweigh the risks) and the availability of alternative treatments for the investigated condition. Thus, research and, eventually, clinical practice are based on previously approved tDCS devices with safety specifications, combined with careful patient selection through inclusion and exclusion criteria guided by medical knowledge, and the subject's ability to understand and consent to the offered intervention.

In most countries, an ethics committee that assesses the adequacy of the protocol in accordance with the Declaration of Helsinki, the principles of bioethics and local legislation, evaluates the research protocol. The researcher, at the approval of the protocol, should follow it without deviations, including the use of the same devices in the same target population.

In Brazil, medical devices can only be used after they have been tested and approved by the ANVISA. In the USA, the FDA is yet to approve the use of tDCS devices, although they can be used "off label," while in the European Union some tDCS devices have already been regulated. The regulation of non-medical tDCS use is, in fact, not possible by the FDA or European agencies, as medical/non-medical use is defined not by the intervention's mechanism of action but rather by its usage intention - thus the marketing strategies of companies advertising tDCS as a device designed to "turbine your memory" or "increase your well-being," denoting non-medical use.

However, the technical divisions of Psychiatry, Neurology or Physical Medicine and Rehabilitation of the 
Brazilian CFM have not yet made any pronouncements about tDCS use. This puts its use for medical purposes in Brazil in a "limbo," where it has been approved by ANVISA, but without any CFM manifestations - contrarily or favorably. The CFM, in Resolution $1982 / 2012$, states that using medications not endorsed by CFM is an ethical infraction. ${ }^{37}$ Thus, the use of tDCS in medical practice in Brazil could be considered an ethical infraction. Nevertheless, CFM rebuttals to specific questions regarding the use of unregulated drugs were generally favorable to their use, considering the principles of non-maleficence and beneficence. In neuromodulation, it is worth remembering that electroconvulsive therapy (ECT) and transcranial magnetic stimulation (rTMS) were only officially regulated by the CFM in 2002 and 2012, respectively, but had been already in clinical use beforehand (notably ECT). In addition, present legislation regulating rTMS use defines parameters that are largely obsolete. ${ }^{38}$

Another issue is the DIY-tDCS movement. There are websites on the Internet, such as www.diytdcs. com, www.totaltdcs.com and www.tdcsplacements.com, that summarize studies in the literature to indicate the "correct assemblage of devices" for the improvement of cognitive functions. In studies about this community, Wexler showed that DIY-tDCS adepts are young, male, and American. Their approach mimics a "scientific" use, by reading data in the literature, doing sessions with controlled parameters, and measuring results using depression and anxiety scales or cognitive tests freely available on the Internet.

In this sense, it seems critical that at least minimal standards of non-medical use should be proposed. ${ }^{21}$ Some authors suggest extending the biomedical model to the "cosmetic" use of neurostimulation devices, such as tDCS. ${ }^{39}$ This regulatory approach may be relevant in that tDCS devices are the same for clinical use and for cognitive enhancement purposes and also because the separating line between "normal" and "pathological" is becoming less and less distinct. In turn, other authors have proposed the creation of an official online forum where the DIY-tDCS community could receive advice and safety guidelines, with the possibility of expert consultation. ${ }^{40}$ This approach is guided by the principle of "harm reduction," and aligns with the demand by tDCS users for guidance and advice. For example, simple guidelines such as the need to use saline solution to humidify the sponges that cover the electrodes, the correct use of elastics for the proper positioning of the electrodes, the maximum dose and duration of a stimulation session, etc., would make the use of the technique much safer. However, this proposal is not without costs, since specialists are needed, unless the proposal calls for pro bono expert participation. In addition, the spontaneous offer of risk management in an unofficial forum could potentially increase the number of tDCS users, with still uncertain results. Moreover, it remains open to question who would be liable in this context - the company that produced the device, the person who used it or the professional who directed its use.

Some authors have also proposed framing the tDCS devices as articles for personal use, without considering them as medical devices, along with a program of awareness and education. To avoid the risks associated with electrode maladjustment or current intensity, user manuals and training could be offered to safeguard the minimum safety regarding the use of tDCS in selected populations, such as adults older than a specific age. At a minimum, any guideline should present the real known risks and benefits of using tDCS, based on the best available evidence. Conversely, the uncertainty about the real long-term efficacy and side effects of using tDCS devices in a real-life setting must be clearly explained by tDCS device manufacturers and other professionals involved in their marketing. The urgency of a standpoint is all the greater, because of the lack of data on the long-term effects of tDCS, conflicting results regarding the effect size of the technique, and its increasingly widespread use for non-medical purposes.

\section{Final considerations}

In addition to the discussion about whether and how tDCS should be regulated, the social and historical contexts that permeate these discussions must be considered. In this sense, Wexler compares the current development of tDCS with medical electrotherapy at the turn of the 19th century (1870-1920). ${ }^{41}$ At that time, electrical stimulation devices were used in the medical community, but especially among laypeople as a form of treatment for various pain and psychological complaints. Just like presently with tDCS, electrotherapy devices were low-cost, portable and could be purchased by mail and used with little or no medical support.

In her review, Wexler noted some phenomena in common between current and past use. For example, the idea of DIY was also present, with magazines teaching how to set up the "medical battery" as well as the idea of doing something "anti-establishment" for example, self-treatment, abdicating the need for a doctor. Finally, the "establishment" (i.e., medical and scientific associations), in the past, was contrary to the "irregular" use of electrotherapy, a trend also observed contemporaneously. 
Wexler also noted three main differences between current and previous use, one of methodological origin (placement of electrodes) and two other quite present currently but rarely mentioned in the past: the question of risk and safety, and use for cognitive enhancement. Interestingly, these questions are at the heart of the discussion about autonomy and safety in neuroethics, reflecting two principles that are not completely harmonic, since there is a limit for individuals to exchange their safety for their autonomy in deciding whether to receive the intervention (the same discussion applies to controlled and illicit drug use, for example).

The issue of different patterns of choice of a society in their health policies and scientific priorities might suggest possible underlying social ideologies. The prevailing ideology of psychiatry as part of medicine should be its scientific spirit, based on the values of independence of thought, criticism, fairness, social responsibility and "faith in science" (i.e., the belief that nature is governed decisively by variable laws).

However, this perspective clashes with the current Zeitgeist, in which the logic of increasing productivity and shortening temporality (sense of time) becomes increasingly prevalent. The term "enjoy life," which could lead to some interesting and creative subjective effects, becomes rather sterile when the idea of "enjoy" is bound with the logic of productivity and accumulation. Thus, a hobby such as playing video games must be improved to the maximum degree by "hacking" the brain.42 Furthermore, ultracompetitive markets, the demand for super-specialization and concurrent unemployment rates lead young people to increasingly look for ways to enhance cognition and decrease fatigue.

The DIY-tDCS movement also finds echoes in the growing demand for empowerment. In fact, the DIY is not limited to tDCS devices. Thus, building a biomedical device following instructions on the Internet to improve sleep and treat depression, for example, regardless of the outcome, is a very enticing idea for the proponents of empowerment.

In this context, the fundamental question is the extent to which psychiatry should depart from its scientific spirit to adjust to the current social values of greater and sometimes reckless productivity, thereby incorporating the cosmetic use of neuromodulation. Between the two possible solutions - total release or total restriction - there is a spectrum of options that can be contemplated. In addition, the decision may vary over time and in different societies and countries. From a medical point of view, however, the guiding principles of neuroethics must be present at all times to ensure the best interest of those who choose to receive electrical stimulation.

\section{Acknowledgments}

Andre R. Brunoni is the recipient of a fellowship award for experienced researchers from Coordenação de Aperfeiçoamento de Pessoal de Nível Superior/Fundação Alexander von Humboldt.

\section{Disclosure}

Lucas Borrione reports personal fees from Libbs Laboratory, Apsen Laboratory, and Ache Laboratory. Andre R. Brunoni reports personal fees from the Neurocare Group, DeltaMedical (Magstim distributor in Brazil), and non-financial support from Soterix Medical.

\section{References}

1. Zhao H, Qiao L, Fan D, Zhang S, Turel O, Li Y, et al. Modulation of brain activity with noninvasive transcranial direct current stimulation (tDCS): clinical applications and safety concerns. Front Psychol. 2017;8:685.

2. Brunoni AR, Ferrucci R, Bortolomasi M, Vergari M, Tadini L, Boggio PS, et al. Transcranial direct current stimulation (tDCS) in unipolar vs. bipolar depressive disorder. Prog Neuropsychopharmacol Biol Psychiatry. 2011;35:96-101.

3. Pereira M, Rafiq B, Chowdhury E, Babayev J, Boo H, Metwaly $\mathrm{R}$, et al. Anodal cerebellar tDCS modulates lower extremity pain perception. NeuroRehabilitation. 2017;40:195-200.

4. Brunoni AR, Ferrucci R, Fregni F, Boggio PS, Priori A. Transcranial direct current stimulation for the treatment of major depressive disorder: a summary of preclinical, clinical and translational findings. Prog Neuropsychopharmacol Biol Psychiatry. 2012;39:9-16.

5. Jwa A. Early adopters of the magical thinking cap: a study on do-it-yourself (DIY) transcranial direct current stimulation (tDCS) user community. J Law Biosci. 2015;2:292-335.

6. Borducchi DM, Gomes JS, Akiba H, Cordeiro Q, Borducchi JH, Valentin LS, et al. Transcranial direct current stimulation effects on athletes' cognitive performance: an exploratory proof of concept trial. Front Psychiatry. 2016;7:183.

7. Looi CY, Duta M, Brem AK, Huber S, Nuerk HC, Cohen Kadosh R. Combining brain stimulation and video game to promote longterm transfer of learning and cognitive enhancement. Sci Rep. 2016;6:22003.

8. tDCSPlacements. DARPA's accelerated learning [web page]. [cited 2017 Jun 06]. http://tdcsplacements.com/placements/ accelerated-learning

9. Wexler A. A pragmatic analysis of the regulation of consumer transcranial direct current stimulation (TDCS) devices in the United States. J Law Biosci. 2015;2:669-96.

10. Fregni F, Nitsche MA, Loo CK, Brunoni AR, Marangolo P, Leite $J$, et al. Regulatory considerations for the clinical and research use of transcranial direct current stimulation (tDCS): review and recommendations from an expert panel. Clin Res Regul Aff. 2015;32:22-35.

11. Figueroa G. Neuroethics: the pursuit of transforming medical ethics in scientific ethics. Biol Res. 2016;49:11.

12. Talsma LJ, Kroese HA, Slagter HA. Boosting cognition: effects of multiple-session transcranial direct current stimulation on working memory. J Cogn Neurosci. 2017;29:755-68. 
13. Sparing R, Thimm M, Hesse MD, Kust J, Karbe H, Fink GR. Bidirectional alterations of interhemispheric parietal balance by non-invasive cortical stimulation. Brain. 2009;132:3011-20.

14. Cattaneo Z, Pisoni A, Papagno C. Transcranial direct current stimulation over Broca's region improves phonemic and semantic fluency in healthy individuals. Neuroscience. 2011;183:64-70.

15. Hauser TU, Rutsche B, Wurmitzer K, Brem S, Ruff CC, Grabner RH. Neurocognitive effects of transcranial direct current stimulation in arithmetic learning and performance: a simultaneous tDCS-fMRI study. Brain Stimul. 2016;9:850-8.

16. Pisoni A, Cerciello M, Cattaneo Z, Papagno C. Phonological facilitation in picture naming: When and where? A tDCS study. Neuroscience. 2017;352:106-21.

17. Kelley NJ, Eastwick PW, Harmon-Jones E, Schmeichel BJ. Jealousy increased by induced relative left frontal cortical activity. Emotion. 2015; 15:550-5.

18. Civai C, Miniussi C, Rumiati RI. Medial prefrontal cortex reacts to unfairness if this damages the self: a tDCS study. Soc Cogn Affect Neurosci. 2015;10:1054-60.

19. Kuehne $M$, Heimrath $\mathrm{K}$, Heinze $\mathrm{HJ}$, Zaehle T. Transcranial direct current stimulation of the left dorsolateral prefrontal cortex shifts preference of moral judgments. PLoS One. 2015;10:e0127061.

20. Mameli $F$, Mrakic-Sposta S, Vergari M, Fumagalli M, Macis M, Ferrucci $R$, et al. Dorsolateral prefrontal cortex specifically processes general - but not personal - knowledge deception: Multiple brain networks for lying. Behav Brain Res. 2010;211:164-8

21. Dubljevic V, Saigle $V$, Racine $E$. The rising tide of tDCS in the media and academic literature. Neuron. 2014;82:731-6.

22. Sellers KK, Mellin JM, Lustenberger CM, Boyle MR, Lee $W_{\text {, }}$ Peterchev AV, et al. Transcranial direct current stimulation (tDCS) of frontal cortex decreases performance on the WAIS-IV intelligence test. Behav Brain Res. 2015;290:32-44.

23. Sarkar A, Dowker A, Cohen Kadosh R. Cognitive enhancement or cognitive cost: trait-specific outcomes of brain stimulation in the case of mathematics anxiety. J Neurosci. 2014;34:16605-10.

24. Mancuso LE, Ilieva IP, Hamilton RH, Farah MJ. Does transcranial direct current stimulation improve healthy working memory? A meta-analytic review. J Cogn Neurosci. 2016;28:1063-89.

25. Horvath JC, Forte JD, Carter O. Quantitative review finds no evidence of cognitive effects in healthy populations from singlesession transcranial direct current stimulation (tDCS). Brain Stimul. 2015;8:535-50.

26. Lefaucheur JP. A comprehensive database of published tDCS clinical trials (2005-2016). Neurophysiol Clin. 2016;46:319-98.

27. Horvath JC, Carter O, Forte JD. Transcranial direct current stimulation: five important issues we aren't discussing (but probably should be). Front Syst Neurosci. 2014;8:2.

28. To WT, Hart J, De Ridder D, Vanneste S. Considering the influence of stimulation parameters on the effect of conventional and highdefinition transcranial direct current stimulation. Expert Rev Med Devices. 2016;13:391-404.
29. Brunoni AR, Ferrucci R, Bortolomasi M, Scelzo E, Boggio PS, Fregni $F$, et al. Interactions between transcranial direct current stimulation (tDCS) and pharmacological interventions in the Major Depressive Episode: findings from a naturalistic study. Eur Psychiatry. 2013;28:356-61.

30. Katz B, Au J, Buschkuehl M, Abagis T, Zabel C, Jaeggi SM, et al. Individual differences and long-term consequences of tDCSaugmented cognitive training. J Cogn Neurosci. 2017:1-11.

31. Aparicio LV, Guarienti $F$, Razza LB, Carvalho AF, Fregni $F$ Brunoni AR. A Systematic review on the acceptability and tolerability of transcranial direct current stimulation treatment in neuropsychiatry trials. Brain Stimul. 2016;9:671-81.

32. Li MS, Chen YY, Hung GC. Rapid onset of hypomania associated with transcranial direct current stimulation in a patient with bipolar depression. Brain Stimul. 2016;9:955-6.

33. Galvez V, Alonzo A, Martin D, Mitchell PB, Sachdev P, Loo CK. Hypomania induction in a patient with bipolar II disorder by transcranialdirectcurrentstimulation(tDCS). JECT. 2011;27:256-8.

34. Steenbergen $L$, Sellaro R, Hommel B, Lindenberger $U$, Kuhn S, Colzato LS. "Unfocus" on foc.us: commercial tDCS headset impairs working memory. Exp Brain Res. 2016;234:637-43.

35. Harari Y. Homo Deus: a brief history of humankind. London: Vintage; 2016.

36. Lavazza A. Can neuromodulation also enhance social inequality? Some possible indirect interventions of the state. Front Hum Neurosci. 2017;11:113.

37. Conselho Federal de Medicina. Resolução CFM no 1.982/2012 [web page]. 2012. [cited 2017 Jun 06]. http://www.portalmedico. org.br/resolucoes/CFM/2012/1982_2012.htm

38. Conselho Federal de Medicina. Resolução CFM no 1.986/2012 [web page]. 2012. [cited 2017 Jun 06]. http://www.portalmedico. org.br/resolucoes/CFM/2012/1986_2012.pdf

39. Maslen H, Douglas $T$, Cohen Kadosh R, Levy N, Savulescu J. The regulation of cognitive enhancement devices: extending the medical model. J Law Biosci. 2014;1:68-93.

40. Fitz NS, Reiner PB. The challenge of crafting policy for do-ityourself brain stimulation. J Med Ethics. 2015;41:410-2.

41. Wexler A. Recurrent themes in the history of the home use of electrical stimulation: Transcranial direct current stimulation (tDCS) and the medical battery (1870-1920). Brain Stimul. 2017; 10:187-95.

42. Wexler A. The social context of "do-it-yourself" brain stimulation: neurohackers, biohackers, and lifehackers. Front Hum Neurosci. 2017;11:224.

\section{Correspondence:}

Andre R. Brunoni

Instituto de Psiquiatria, Hospital das Clínicas

Av. Dr. Ovidio Pires de Campos 785, $2^{\circ}$ andar, Ala Sul

01060-970 - São Paulo, SP - Brazil

E-mail: brunowsky@gmail.com 